\title{
A Recovery-Oriented Approach for an Acute Psychiatric Ward: Is It Feasible and How Does It Affect Staff Satisfaction?
}

\author{
Franziska Rabenschlag • Albrecht Konrad • Sebastian Rueegg • \\ Matthias Jaeger
}

Published online: 4 December 2013

(C) Springer Science+Business Media New York 2013

\begin{abstract}
To evaluate professionals' attitudes to recovery and coercion, as well their satisfaction with working conditions before and after the implementation of a recoveryoriented ward concept on an admission ward. Longitudinal study design with two measurement times of the study sample, with a control group assessed at study end. Evaluating the implementation of the recovery concept, attitudes towards recovery, coercion, perceptions of the ward and working satisfaction were assessed with questionnaires and computed using Chi square and ANOVA variance analyses. The members of the intervention ward $(n=17)$ did not differ from the control group $(n=21)$, except that control group members were younger. The recovery-orientation of the study ward (ROSE questionnaire) increased significantly (alpha level $=0.05$ ) from study begin to study end $(p=0.003)$, and compared to the control group $(p=0.002)$. The attitudes towards coercion did not change significantly in the intervention group, but did so compared to the control group. The contentedness (GMI) and the satisfaction with working conditions $(\mathrm{ABB})$ of the intervention group members compared to control group was significantly higher (GMI: $p=0.004$, ABB subscale working conditions: $p=0.043$, satisfaction: $p=0.023$ ). The study indicates that recovery-oriented principles can be implemented even in an acute admission ward, increasing team satisfaction with work, while attitudes towards coercion did not change significantly within this single-unit project.
\end{abstract}

Keywords Recovery $\cdot$ Psychiatric admission ward · Working satisfaction

F. Rabenschlag ( $\square)$

Psychiatric University Clinics, Wilhelm Klein-Strasse 27, 4012 Basel, Switzerland

e-mail: franziska.rabenschlag@upkbs.ch

A. Konrad · S. Rueegg · M. Jaeger

Psychiatric University Hospital, Zurich, Switzerland 


\section{Background}

Along with a growing demand for more patient-orientated, individual treatment involving the people concerned there has been a change in treatment processes and structures in psychiatric institutions towards recovery-oriented practice [1,2]. The recovery-oriented approach of mental health services stands for a change in roles, values and attitudes on the part of health professionals: from an expert-role to a supporting and accompanying partnership; from a paternalistic, rather deficit-orientated attitude towards a psychiatric service where structures and professional attitudes are transparent, health-orientated, and clientautonomy enhancing [3, 4]. A patient-oriented treatment offers transparent forms of communication, for example accordant decision-making-models [5], and the fostering of an optimistic, hopeful and supportive attitude [6]. The consideration of the users' perspective in the context of structures and processes is an important element of the concept of recovery $[7,8]$, which in the context of mental illness therefore accounts not only for the attitude of professionals, but also for the treatment structures of health services [4, 9]. Moreover, psychiatric services with a recovery-oriented treatment offer on the one hand evidence-based treatments, and on the other, treatments that are subjectively felt to be effective [4].

The implementation of the recovery concept in health services is discussed controversially $[7,10,11]$, and there are inconsistent results concerning instruments for measuring a service's level of recovery orientation [12]. To support the implementation of a recovery-oriented practice, regional as well as national manuals were developed, for example the guidelines of the American Association of Community Psychiatrists [13]. In their qualitative analysis of 15 international practice guidelines Le Boutillier et al. [11] structured the recovery-relevant themes into four key domains: promoting citizenship, organizational commitment, supporting personally defined recovery, and working relationship. But more and more discussion has focussed on one of the largest stumbling blocks on the way towards recovery orientation in an acute inpatient setting, namely, the reality of involuntary admissions and measures of constraint [14, 15]. Both are mainly a task of psychiatric hospitals rather than of psychiatric services in community settings. Consequently, implementation of recovery-oriented practice is better known in outpatient settings and has been more often the target of evaluations there [16]. The staff's recovery orientation has been shown to be higher in outpatient settings than in inpatient services [17-19]. But it has also been shown that a recovery-relevant attitude of staff members can be altered positively with 2 days' training $[20,21]$ or even with a single training session [22].

The aim of this study was to evaluate professionals' attitudes towards recovery and coercion as well as their satisfaction with working circumstances and the ward atmosphere before and after the implementation of a recovery-oriented ward concept on an admission ward. The recovery-oriented approach used here was developed following the guidelines of the American Association of Community Psychiatrists [13]. Structures of reports and consultations were changed, an individual goal setting was implemented, and the staff of the ward was informed and trained concerning the recovery concept.

\section{Implementation of Recovery-Orientation in an Admission Ward}

At the beginning of the study period (September 2011) the structure of reports and ward rounds were changed with the intention of increasing transparency by involving patients 
and their family members. In detail the timeframe of reports without the patients were reduced by $50 \%$ to 15 -min daily briefings on workday mornings. Individual goal setting and therapy planning for the stay in the psychiatric service was implemented, written together by members of the interdisciplinary team and the patient. The written planning was evaluated together regularly. The total time spent on staff discussion without the presence of the patient concerned was reduced from 210 to 75 min per week. Besides these structural modifications the members of the multidisciplinary study ward team were trained in theoretical and practical issues concerning recovery. Furthermore an external expert on recovery orientation (a psychologist) conducted a three-day training, which consisted of relevant aspects and questions in the implementation phase.

The intervention ward is an acute admission ward. Its door is often open but closeable when necessary. The ward comprises one- to three-bed rooms and one seclusion room. It can treat up to 16 patients with any kind of a psychiatric diagnosis. In the year 2012, the largest groups of patients were suffering from a schizophrenic disorder (44\%), a substance related disorder $(25 \%)$ or an affective disorder (16\%). The average duration of stay was 25 days, and $50 \%$ of the patients were admitted involuntarily.

\section{Methods}

\section{Study Design and Sample}

A longitudinal study design was chosen, with two measurement times ( $t_{0}$ before the implementation, $t_{1}$ after recovery training) of the study sample over one year, from September 2011 to October 2012. A control group was recruited as a convenience sample from three control wards due to their comparability of patients, number of beds and seclusion rooms as well their professionals, and assessed at $t_{1}$ using the same instruments.

The intervention group sample consists of the multidisciplinary team members of the intervention ward, which involves nurses (=15 full-time staff), physicians (300\%), social workers $(40 \%)$ and occupational-, vocational- and physio-therapists (120\%). There was no criterion for exclusion. After receiving information about the study the participants signed written informed consent. Participation was voluntary. Each participant could randomly choose a personal number that was blinded to other participants as well as to the researchers. The number remained valid during the entire study. The ethics commission of the concerning state approved the study.

Instruments

To measure the parameters related to the recovery relevant attitudes of the team members the following instruments were used. Their internal consistency was assessed with Cronbach's alpha. In order to measure their contentedness with the work the Good Milieu Index, GMI [23], containing 5 items on a 4-point Likert scale (ranging from 0 to 3 , alpha $=0.675$ ), and the ABB [24], also with 5 items, 2 of them on a 4-point Likert scale (ranging from 0 to 3 ) and 3 of them on a 7-point vision scale were used. To assess the level of the recovery attitudes the Recovery Attitudes Questionnaire, RAQ-7 [25] with 7 items on a 5-point Likert scale (ranging from 0 to 4 , alpha $=0.653$ ) was chosen. The two subscales of RAQ-7 (Recovery is possible, Recovery is difficult and needs faith) were not further computed due to their poor alpha-values (alpha <0.6), [26]. In order to assess attitudes towards coercion the Staff Attitude Coercion Scale SACS [27] with 15 items on a 
5-point Likert scale was used (alpha $=0.648$ ). Here too, its 3 subscales (coercion as offending, coercion as care and security, and coercion as treatment) were not further included (alpha $<0.6$ ). For the perception of the wards' atmosphere the Essen Climate Evaluation Scheme, Essen CES [28] with 3 subscales (patient cohesion, safety, and therapeutic hold) and 17 items on a 5-point Likert scale (range from 0 to 4 , alpha $=0.714$ ), and of the wards' level of recovery orientation the Recovery Oriented Service Evaluation, AACP Rose [29] with 4 subscales (administration, treatment, supports, organizational culture), and 46 items on an 5-point Likert scale were used (range from 0 to 4 , alpha $=0.963)$. The psychosocial burden of the participants was assessed using the Symptom Check List SCL-10 [30] with 10 items on a 5-point Likert scale (from 0 to 4, alpha $=0.732$ ). Additionally, sociodemographic data, i.e. age, sex, working experience (years, type of psychiatric settings), absence (illness, holidays) were collected.

\section{Statistical Analyses}

To reveal whether the attitudes of the members of the intervention group change over study time $\left(t_{0}-t_{1}\right)$, and differ compared to the control group, categorical demographic variables were computed with Chi square test. The differences for continuous variables, assessed by means, were computed with ANOVA variance analyses. The distributions of the scales were tested with Q-Q-plots, skewness and kurtosis. They were normally distributed, except one out of two subscales of questionnaire RAQ 7, which was analyzed using non-parametric tests. A level of significance was set at $p=0.05$ (two-tailed). All data were analyzed with the SPSS, Version 20 (Statistical Package for the Social Sciences, IBM Corporation, 2011).

\section{Results}

\section{Demographics}

The study sample consisted of staff members of the intervention ward (intervention group) and the control sample of staff members of three comparison wards (control group). The study sample at $t_{0}$ consisted of 16 , and at $t_{1}$ of 17 respondents, of whom 7 participated at both time points. The control sample comprised 21 participants (Table 1).

The demographic variables of the staff-members from the intervention ward did not change significantly from the beginning to the end of the study time one year later. Only the variables absence due to holidays or illness showed significant differences between the groups, indicating that the team members were more often absent due to holidays and/or illness at the end of the study than at its beginning (Table 1).

The staff members of the intervention group and the members of the control group did not differ significantly in the demographic variables sex, profession, years of professional experience, absence due to holidays or illness, and previous work setting in a psychiatric institution (Table 1). They did differ significantly concerning the variable age, since the control group had more young staff members aged between 18 and 25 years, and the variable days of work presence (Table 1). Almost none of the professionals of any ward had previous experience in working in an outpatient setting nor did they have experience in a day hospital, home care or other setting. 


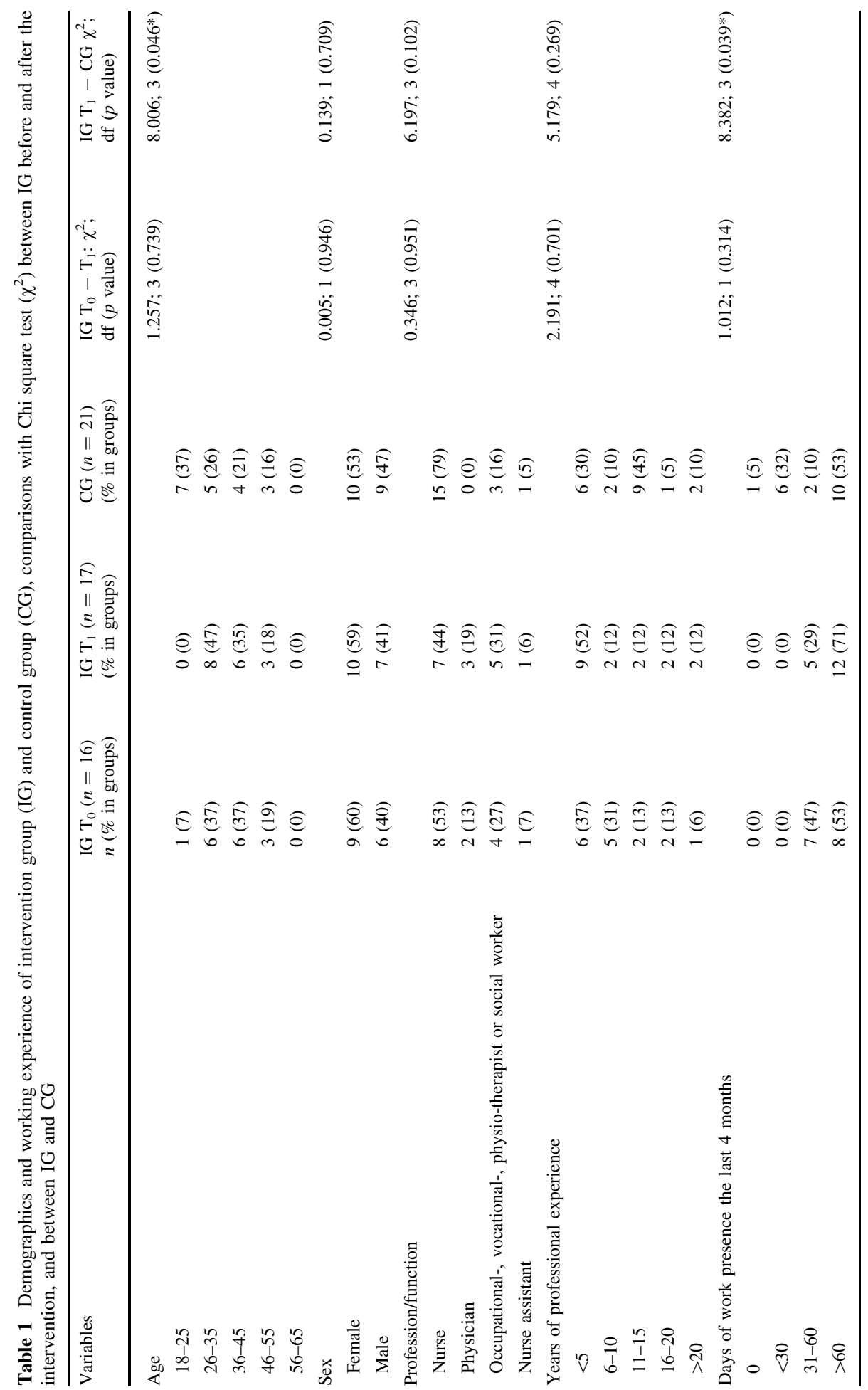




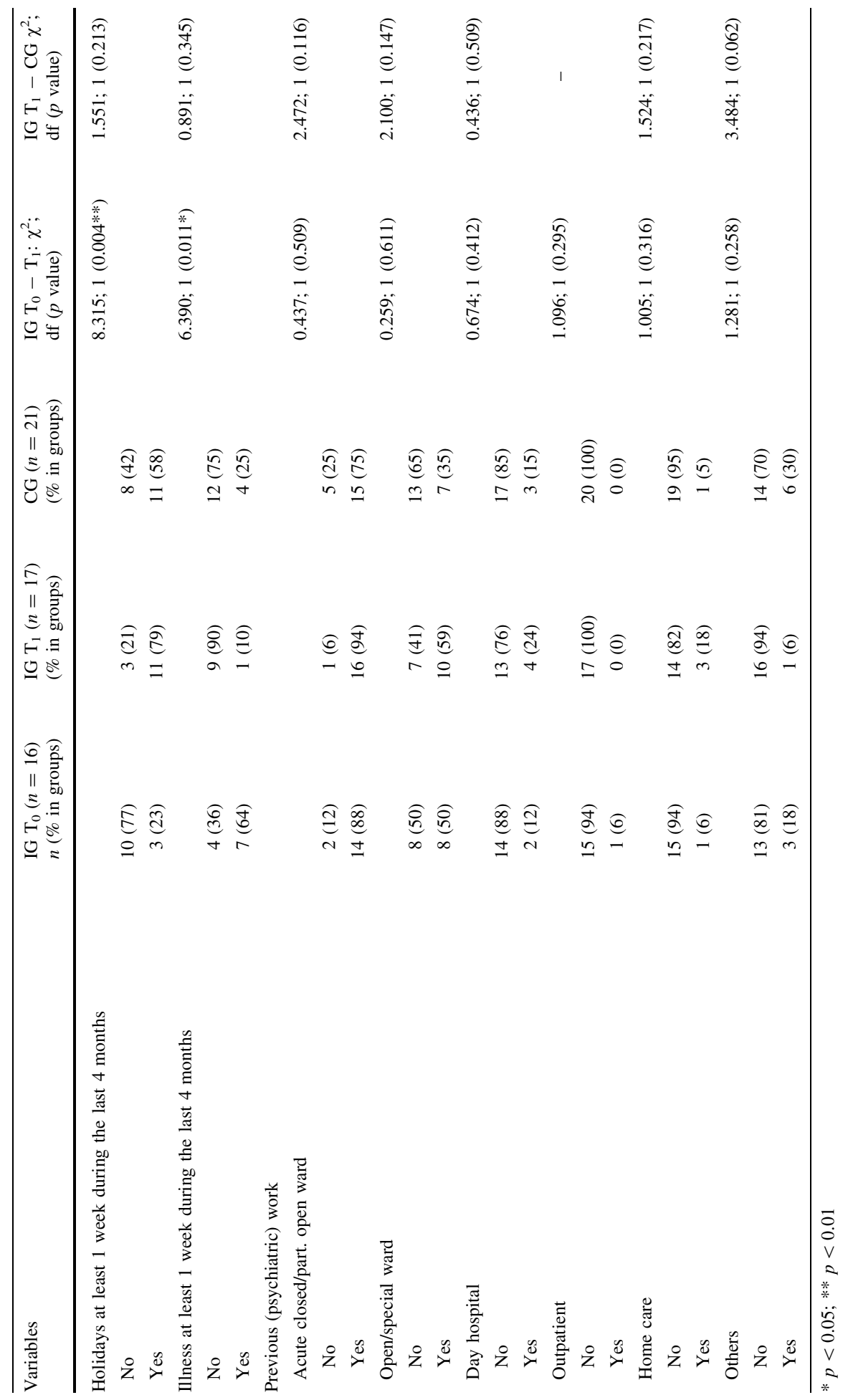


Results of Intervention Group Evaluation at Start Compared to End of the Study

The assessment of the recovery-orientation of the study ward by the members of the intervention group at the beginning $\left(\mathrm{t}_{0}\right)$ of the study compared to them at the end of study $\left(t_{1}\right)$ revealed that the ward gained in its recovery orientation: the means of the overall questionnaire ROSE increased significantly, as did its subscale,treatments',,supports' and,organizational culture'. The subscale,administration' did not change (Table 2). Satisfaction with the ward as a workplace and with the work itself tended to improve among staff during the study (GMI overall, several items of GMI and ABB; Table 2). Attitudes towards recovery (RAQ-7) as well towards coercion (SACS) did not change significantly over the study time. Also, perception of ward atmosphere (CES) did not alter significantly from the beginning of the study to the measurement point a year later.

The differences in the single items of the ROSE scale before and after the study time were compared (ANOVA) in order to shed light on details of the ward changes during the study intervention (Table 3). Single items of the subscale, administration' were omitted in Table 3 because the subscale did not change significantly. Most items of the other subscales significantly improved during the study period (Table 3).

\section{Results of Intervention Group Compared to Control Group}

A number of instruments used in the evaluation revealed significant differences between the intervention group and the control group (Table 2). The attitudes towards recovery (RAQ 7) in general were more positive in the intervention group. Like the assessment of the ward's recovery-orientation (ROSE total score and subscales treatments, supports and organizational culture) was higher on the intervention ward than on the control wards, with the exception of the subscale administration (Table 2). Two of three subscales of the questionnaire CES showed a more positive perception of the intervention ward's atmosphere. The contentedness of the members of the intervention ward compared to control wards was significantly higher. Also satisfaction with working conditions was significantly higher (GMI, ABB; Table 2).

\section{Discussion}

The study at hand aimed to analyse the implementation of a recovery-oriented concept within an acute inpatient setting. Since recovery-oriented services are more commonly established in outpatient settings the goal of this clinical research was to evaluate if and how the implementation of a recovery-oriented concept on an admission ward would be reflected in various measures assessed by staff of different professions.

The main results are that staff rated the recovery orientation of the study ward after the intervention higher on all counts than before the intervention as well as compared to the control group. Moreover, staff rated some aspects of work satisfaction higher both after the implementation of the new concept and compared to the control wards. The implementation of the concept had no influence on attitudes towards (personal) recovery itself or towards coercion. However, the intervention group had more positive attitudes towards recovery as well as more positive views on ward atmosphere after the intervention compared to the control group.

The intended alterations on the ward towards recovery-orientation are reflected in professionals' evaluation of the ROSE questionnaire. Considerable changes in the desired 


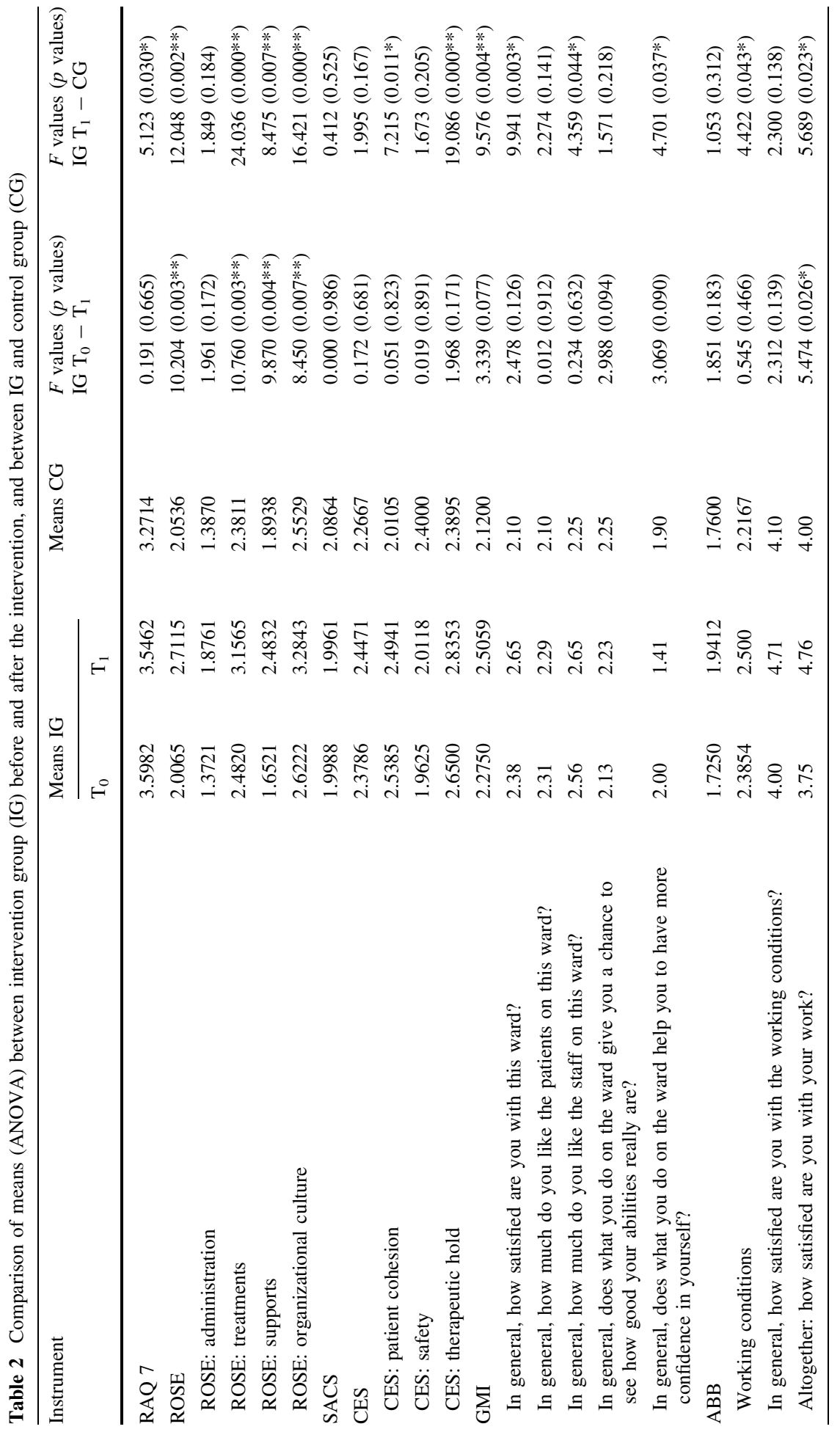




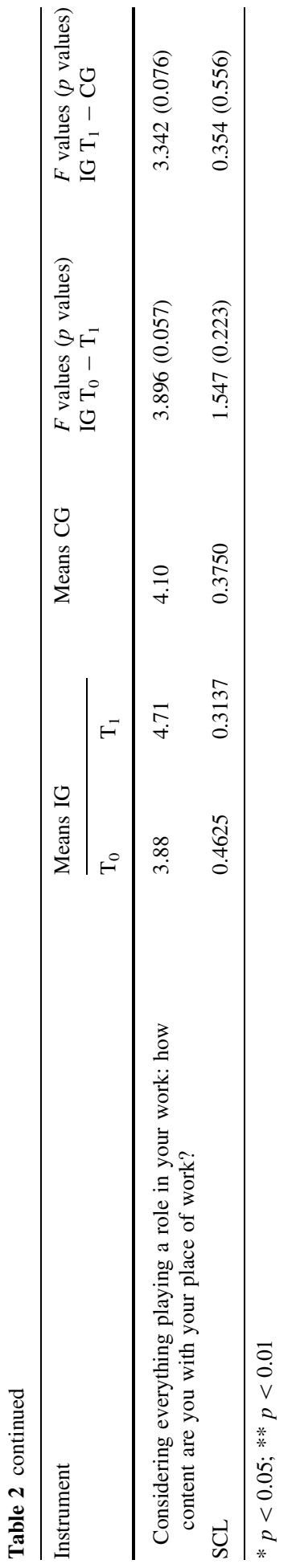




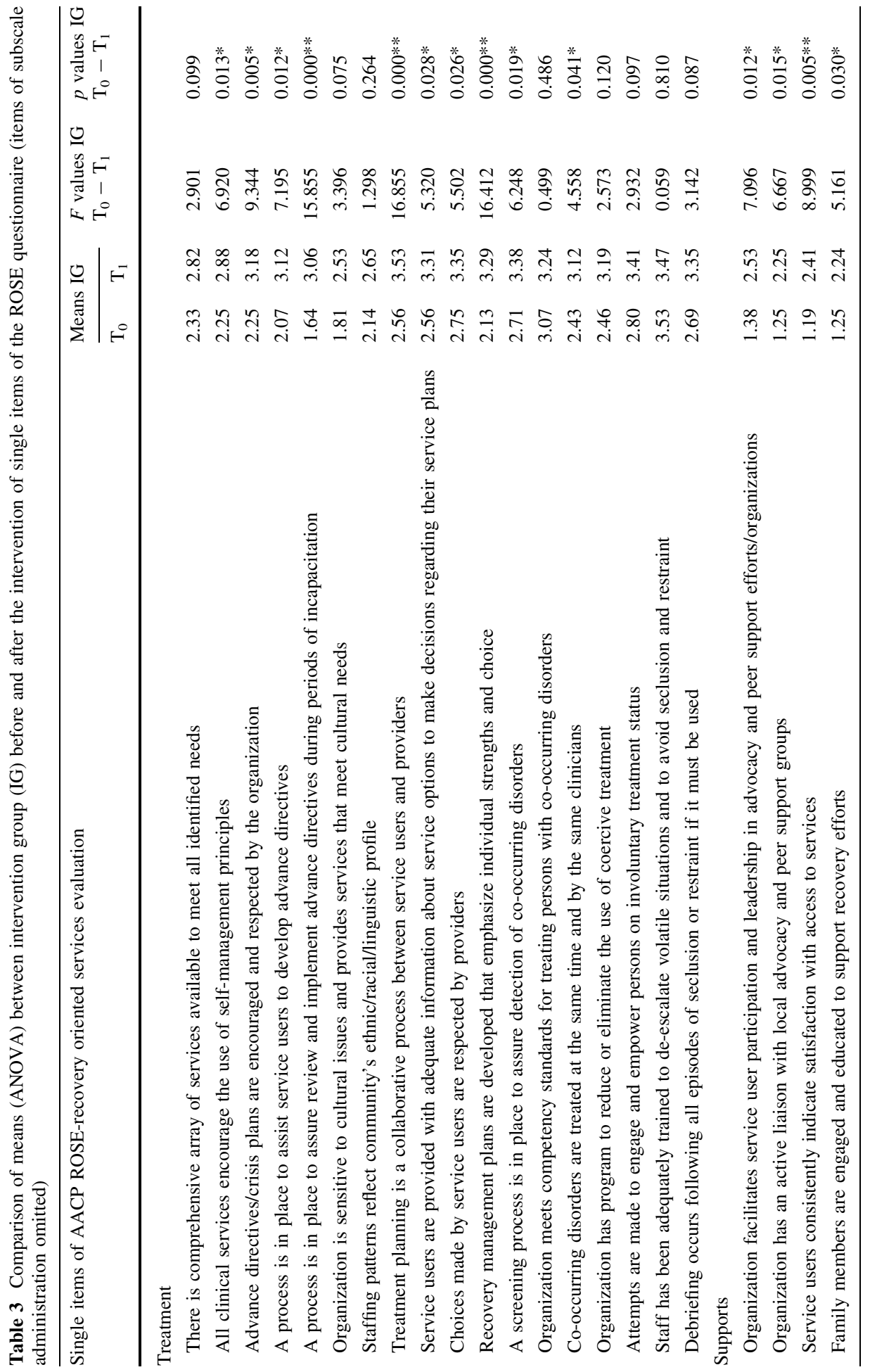




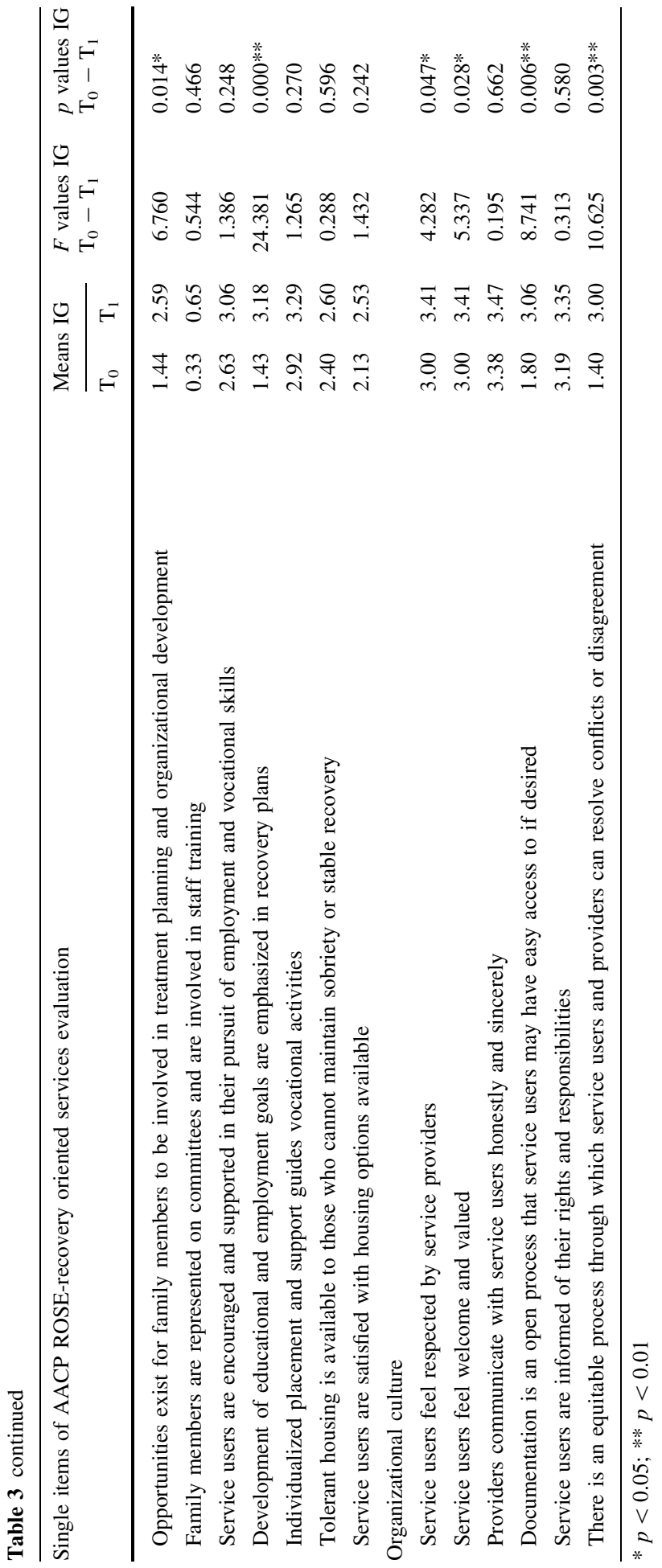


direction were determined concerning treatments and supports as well as related to the organizational culture of the ward. This is consistent with two out of three domains of Sowers' guideline for recovery-oriented services, i.e. treatment and supports [13]. No substantive changes were found concerning the domain of administration involving, e.g., an organizational commitment to foster recovery, continuous improvement in quality management, or the involvement of service users on various institutional levels. Because the implementation of the recovery-oriented approach was limited to one ward as a pilot project without explicit implementation of the notion of recovery on an institutional level this result was expected. Le Boutillier et al. [11], with their qualitative analysis, extracted organizational commitment as one out of four practice domains for guidance, and discussed the importance of the involvement of a whole system. This may lead to a more sustainable shift towards the recovery orientation of a psychiatric service, but this study already shows positive changes of clinical, hands-on alterations concerning the every-day treatments and the therapeutic attitude on a single-unit-level (ROSE).

Along with the implementation of the concept in this study ward there was higher work satisfaction in general, and in particular, a better opportunity to deploy their own abilities at work as well as greater self-confidence. This refers to a study published in 2012, which showed a higher level of work satisfaction and a lower level of exhaustion among 114 case managers working in recovery-oriented community mental health services [31] as well to a study published in 2004, which highlighted a correlation between self-realization and satisfaction with the ward. Although the ward atmosphere as rated on the CES did not change in the course of this study, it is significantly more positive compared to the control wards in the aspects,therapeutic hold' and,patient cohesion' but not concerning,safety' [32].

Attitudes towards recovery did not change among staff of the intervention ward during the study, although these individuals held more positive attitudes towards recovery than did staff members on the control wards. However, attitudes concerning recovery were already positive among staff of the study ward at study begin, pointing to an openness towards recovery orientation in the first place.

The attitudes towards coercion did not significantly change among staff of the study ward during the study nor compared to the control group. The latter result is in line with literature that implies that the attitude towards coercion needs time to be influenced [33] as well as having strong leadership of service management [34]. Perhaps this result indicates the limitations of a single-unit project and could be influenced more distinctly if the reduction or elimination of seclusion and restraint were the target of an institution as a whole. It is understandable that an admission ward as a single unit in a large institution endeavours to find the best way to deal with existing seclusion and restraint, but is not able to eliminate them [34]. Even so, the study ward at hand has more than the half of their patients admitting involuntarily. A care research group called this challenge of acute psychiatric wards as caring approaches between "bulldozer and ballet dancer" [35]. The authors recommend further reflection on the integration of both, meaning that a more paternalistic approach with the target of keeping wards ordered and safe would have its place were it to be done in a professional way like the person-centred approach.

The study at hand has several limitations. Some minor differences between the study groups were detected that might have an influence on the results. Most demographic variables of the intervention group did not differ before and after the intervention, but team members were significantly more often absent due to holidays and illness at study end. This might be explained by the second measurement time point being at the end of the main summer holidays and possibly having an impact on the assessment of work satisfaction, 
ward atmosphere, and attitudes as rather subjective measures but less likely on the evaluation of the ROSE questionnaire that relies on objective sources. However, the absent difference between groups on the SCL as an indicator of personal stress and burden due to psychological symptoms does not support this assumption. Again, this does not apply to the ROSE questionnaire that assesses factual conditions of a ward or institution. As a further limitation, the small sample size has to be considered. This is supposedly related to the high turnover of staff, leading to an overlap of the subsamples before and after the interventions of less than $50 \%$. The strength of the study lies in the evaluation of a naturalistic clinical project with a longitudinal study design and the inclusion of a control group [36].

\section{Conclusions}

The implementation of a person- and recovery-oriented approach on an acute admission ward (with a seclusion room and closed ward door as factors that seem at first sight to contradict a recovery-oriented setting) was achieved in the present project and seems to be possible in general. Consequently treatments, supports and organizational culture as rated by staff members shifted to a recovery-oriented approach. Satisfaction with work increased while attitudes did not significantly change during the limited study period of one year. The present study thus indicates that it is clearly worth the effort for interdisciplinary teams on acute psychiatric wards to deal with the subject of therapeutic attitudes and approaches towards their patients. Recovery-oriented principles can be implemented even in a treatment setting that is traditionally beyond the scope of the recovery movement.

Conflict of interest The authors declare that they have no conflict of interest.

\section{References}

1. Shelton D: The President's New Freedom Commission on Mental Health: Significance for children. Journal of Pediatric Nursing 18(3):203-205, 2003.

2. Slade M: Everyday solutions for everyday problems: how mental health systems can support recovery. Psychiatric Services (Washington, DC) 63(7):702-704, 2012.

3. Farkas M, Gagne C, Anthony WA, Chamberlin J: Implementing recovery oriented evidence based programs: Identifying the critical dimensions. Community Mental Health Journal 41(2):141-158, 2005.

4. Resnick S, Fontana A, Lehman A, Rosenheck R: En empirical conceptualization of the recovery orientation. Schizophrenia Research 75:119-128, 2005.

5. Camann MA: The psychiatric nurse's role in application of recovery and decision-making models to integrate health behaviors in the recovery process. Issues in Mental Health Nursing 31(8):532-536, 2010.

6. Jacobson N, Greenley D: What is recovery? A conceptual model and explication. Psychiatric Services 52(4):482-485, 2001.

7. Bellack AS: Scientific and consumer models of recovery in schizophrenia: Concordance, contrasts, and implications. Schizophrenia Bulletin 32(3):432-442, 2006.

8. Marshall S, Crowe TP, Oades LG, Deane FP, Kavanagh D: A review of consumer involvement in evaluations of case management: Consistency with a recovery paradigm. Psychiatric Services 58(3):396-401, 2007.

9. Amering M, Schmolke M: Recovery in mental health: reshaping scientific and clinical responsibilites. Chichester, Wiley-Blackwell, 2009.

10. Rogers ES, Kash-MacDonald M, Brucker D: Systematic review of peer delivered services literature 1989-2009. Boston: Boston University, Sargent College, Center for Psychiatric Rehabilitation, 2009. http://www.bu.edu/drrk/research-syntheses/psychiatric-disabilities/peer-delivered-services/. Accessed 29 Nov 2013. 
11. Le Boutillier C LM, Bird VJ, Davidson L, Williams J, Slade M: What does recovery mean in practice? A qualitative analysis of international recovery-oriented practice guidance. Psychiatric Services 62(12):1470-1476, 2011.

12. Williams J, Leamy M, Bird V, Harding C, Larsen J, Le Boutillier C, et al.: Measures of the recovery orientation of mental health services: systematic review. Social Psychiatry and Psychiatric Epidemiology 47(11):1827-1835, 2012.

13. Sowers W: Transforming systems of care: the American Association of Community Psychiatrists guidelines for recovery oriented services. Community Mental Health Journal 41(6):757-774, 2005.

14. Jaeger M, Hoff P: Recovery: Conceptual and ethical aspects. Current Opinion in Psychiatry 25(6):497-502, 2012.

15. Ashcraft L, Bloss M, Anthony WA: Best practices: The development and implementation of "no force first" as a best practice. Psychiatric Services 63(5):415-417, 2012.

16. Lloyd-Evans B, Slade M, Jagielska D, Johnson S: Residential alternatives to acute psychiatric hospital admission: Systematic review. The British Journal of Psychiatry : The Journal of Mental Science 195(2):109-117, 2009.

17. Tsai J, Salyers MP: Recovery orientation in hospital and community settings. The Journal of Behavioral Health Services and Research 37(3):385-399, 2008.

18. Tsai J, Salyers MP: Recovery orientation in hospital and community settings. The Journal of Behavioral Health Services and Research 37(3):385-399, 2010.

19. Salyers MP, Tsai J, Stultz TA: Measuring recovery orientation in a hospital setting. Psychiatric Rehabilitation Journal 31(2):131-137, 2007.

20. Salgado JD, Deane FP, Crowe TP, Oades LG: Hope and improvements in mental health service providers' recovery attitudes following training. Journal of Mental Health 19(3):243-248, 2010.

21. Crowe TP, Deane FP, Oades LG, Caputi P, Morland KG: Effectiveness of a collaborative recovery training program in Australia in promoting positive views about recovery. Psychiatric Services 57(10):1497-1500, 2006.

22. Tsai J, Salyers MP, McGuire AB: A cross-sectional study of recovery training and staff attitudes in four community mental health centers. Psychiatric Rehabilitation Journal 34(3):186-193, 2011.

23. Moos R: Evaluating Treatment Environments: A Social Ecological Approach, 2nd edn., New York, London, Sydney, Toronto, Wiley, 1997.

24. Neuberger O, Allerbeck M: Messung und Analyse von Arbeitszufriedenheit. Erfahrungen mit dem "Arbeitsbeschreibungsbogen ABB". Bern, Huber, 1978.

25. Borkin JR: Recovery attitudes questionnaire: Development and evaluation. Psychiatric Rehabilitation Journal. 24(2):95-102, 2000.

26. Jaeger M, Konrad A, Rueegg S, Rabenschlag F: Measuring recovery: Validity of the "Recovery Process Inventory" and the "Recovery Attitudes Questionnaire". Psychiatry Research. 210(1):363-367, 2013.

27. Husum TL, Finset A, Ruud T: The staff attitude to coercion scale (SACS): Reliability, validity and feasibility. International Journal of Law and Psychiatry 31(5):417-422, 2008.

28. Schalast N: A short questionnaire for assessing the social climate on forensic psychiatric wards. Psychiatrische Praxis 35(4):175-181, 2008.

29. American Association of Community Psychiatrist (AACP): AACP ROSE: Recovery Oriented Services Evaluation. In: Campbell-Orde Th, Chamberlin J, Carpenter J, Leff HS (Eds) Measuring the Promise: A Compendium of Recovery Measures. Cambridge, Human Services Research Institute, 2005.

30. Nguyen TD, Attkisson CC, Stegner BL: Assessment of patient satisfaction: development and refinement of a service evaluation questionnaire. Evaluation and Program Planning 6(3-4):299-313, 1983.

31. Kraus SW, Stein CH: Recovery-oriented services for individuals with mental illness and case managers' experience of professional burnout. Community Mental Health Journal 49(1):7-13, 2013.

32. Rossberg JI, Eiring O, Friis S: Work environment and job satisfaction. A psychometric evaluation of the Working Environment Scale-10. Social Psychiatry and Psychiatric Epidemiology 39(7):576-580, 2004.

33. Ashcraft L, Anthony W: Eliminating seclusion and restraint in recovery-oriented crisis services. Psychiatric Services 59(10):1198-1202, 2008.

34. Scanlan JN: Interventions to reduce the use of seclusion and restraint in inpatient psychiatric settings: what we know so far a review of the literature. International Journal of Social Psychiatry 56(4):412-423, 2010.

35. Bjorkdahl A, Palmstierna T, Hansebo G: The bulldozer and the ballet dancer: aspects of nurses' caring approaches in acute psychiatric intensive care. Journal of Psychiatric and Mental Health Nursing 17(6):510-518, 2010.

36. Slade M, Leamy M, Bacon F, Janosik M, Le Boutillier C, Williams J, et al.: International differences in understanding recovery: systematic review. Epidemiology and Psychiatric Sciences 21(4):353-364, 2012. 


\section{Author Biographies}

Franziska Rabenschlag is working as a nurse scientist at the Psychiatric University Clinics in Basel, Switzerland. Her topics are Public Mental Health, Recovery and Peer involvement and the research transfer to practice (evidence based practice). She is a health and nurse expert and has a PhD in Public Health.

Albrecht Konrad (15.02.1968) is an occupational therapist (MScOT), specialized on people with mental health problems who wants to return back to (paid) work. He is working as the line-manager of the vocational therapy department at the Psychiatric University Hospital in Zurich. He is involved in different work rehabilitation programs and targets to integrate the concept Recovery into the vocational training program which is based on occupational therapy theory.

Sebastian Rueegg RN (19.11.1980) is a mental health nurse $(\mathrm{BScN})$ working as a nursing expert on a acute unit at the Psychiatric University Hospital in Zurich. He's responsibilities are for example the implementation of a recovery oriented treatment concept in the clinical setting and the development of strategies to prevent aggression and coercion. His further concerns and efforts are in developing a future approach to provide transitional care and after care treatment.

Matthias Jaeger MD is a Specialist in Psychiatry and Psychotherapy at the Department for Social and General Psychiatry of the Psychiatric University Hospital in Zurich. He works as senior physician on an acute psychiatric ward. His research interests are mental health services research, health economics, subjective perspectives of psychiatric patients, aggression and coercion, and recovery concepts. 